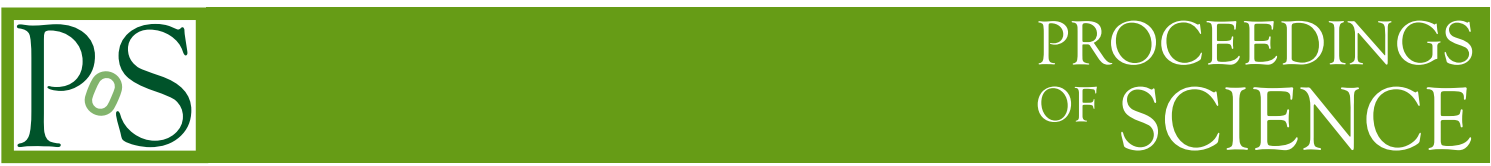

\title{
Study of Physics Performances at Muon Collider
}

\author{
Nazar Bartosik*, Nadia Pastrone
}

INFN Sezione di Torino, Torino, Italy

Alessandro Bertolin, Alessio Gianelle, Lorenzo Sestini

INFN Sezione di Padova, Padova, Italy

\section{Massimo Casarsa}

INFN Sezione di Trieste, Trieste, Italy

\section{Francesco Collamati}

INFN Sezione di Roma, Roma, Italy

\section{Alfredo Ferrari}

CERN, Geneva, Switzerland

\section{Anna Ferrari}

HZDR, Dresden, Germany

\section{Donatella Lucchesi}

University of Padova and INFN Sezione di Padova, Padova, Italy

\section{Nikolai Mokhov, Sergei Striganov}

Fermilab, Batavia, Illinois, U.S.A

\section{Paola Sala}

INFN Sezione di Milano, Milano, Italy

Muon Collider is a promising option for the next generation high-energy collider, possessing very low radiation losses due to synchrotron radiation. Treatment of the beam-induced background is one of the most critical issues in such a machine. Since the muon beams must be very intense to reach high luminosity, the muons decay products and subsequent particles from secondary interactions with the environment can reach the interaction point, limiting the physical performance of the detector. This talk presents a reconstruction strategy for a benchmark process, H->bb-bar, in the presence of the beam-induced background.

XXIX International Symposium on Lepton Photon Interactions at High Energies - LeptonPhoton2019 August 5-10, 2019

Toronto, Canada

\footnotetext{
* Speaker.
} 


\section{Introduction}

In the view of no convincing signature of New Physics observed at the LHC, the HEP community is actively discussing the possible future machine that would open the widest range of possibilities to move the research in particle physics forward. Well established candidates for the LHC successor are hadron-hadron colliders with their high centre-of-mass energy and electron-positron colliders with their clean collision environments. Other possible technologies are investigated in order to answer to the demand for a universal and efficient machine. In this light the muon collider is a very attractive choice, since it possesses both multi-TeV centre-of-mass-energy reach of hadron machines combined with the clean final state of lepton colliders, delivered with minimal losses due to the synchrotron radiation. A muon collider concept faces a number of technological challenges, which are being studied by different groups as summarised in the input from the muon collider (MC) working group to the European strategy update [1].

\section{Beam-induced background}

One of the most critical experimental issues at a muon collider is the limited lifetime of the muons in the beam, which leads to a stream of secondary and tertiary particles arriving to the interaction region (IR). Exact rate and composition of this beam-induced background depends on the layout of the accelerator around the interaction point (IP), experimental hall, detector, etc. Until now the only detailed study of such background has been performed by the MAP collaboration [2], and resulted in the specific design of the machine-detector interface (MDI) [3, 4] featuring a pair of cone-shaped tungsten shields (nozzles) in vicinity of the IP. This study starts from the latest results obtained by the MAP collaboration using their IR and MDI optimized for $1.5 \mathrm{TeV}$ centre-of-mass energy [3] shown in Fig. 1 (a), as well as the realistic sample of background particles arriving to the detector during a single bunch crossing simulated with the MARS15 software [5], which has the particle composition presented in Fig. 1 (b).

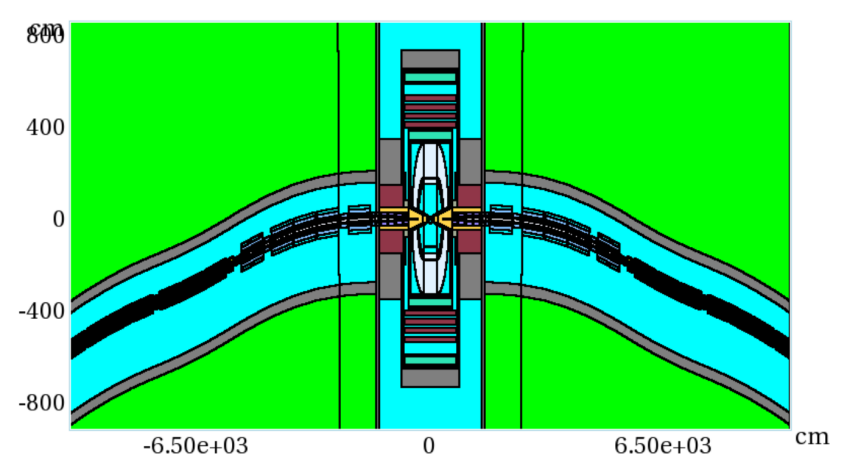

(a)

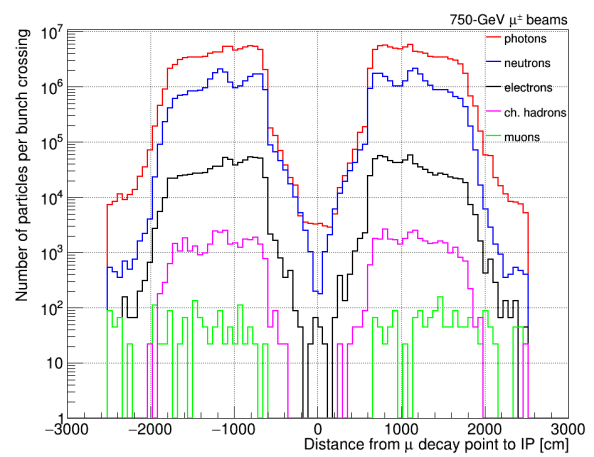

(b)

Figure 1: (a) Illustration of the model used in the MARS15 simulation in a range of $\pm 100 \mathrm{~m}$ around the IP. It includes the machine components in the tunnel, detector and shielding nozzles, which are shown in yellow inside the detector [3]. (b) Particle composition of the beam-induced-background as a function of muon-decay distance from the IP for the case of a $1.5 \mathrm{TeV}$ collider. 


\section{Detector model}

The detector model used in this study is inherited from the previous studies by the MAP collaboration and features vertex and tracking silicon pixel detectors with $20 \mu \mathrm{m}$ and $50 \mu \mathrm{m}$ pitch respectively [6]. The tracking detector is surrounded by a dual readout calorimeter based on the ADRIANO design [7] immersed inside a solenoidal magnetic field of 3.57 T. The outer part of the detector includes a muon spectrometer, which is not simulated in this study. Full simulation of the detector response is performed with the software based on the ILCRoot framework [6] and takes into account sensor efficiency and noise, saturation effects, light attenuation in crystals, etc.

\section{Detector performance}

The number of background particles arriving to the tracking detector in a single bunch crossing would make the track reconstruction with all registered hits impossible. Exploiting the difference in time of arrival between the background particles and the particles originating from the IP it is possible to significantly reduce the occupancy in the tracker. Effectiveness of this method was estimated by assuming $50 \mathrm{ps}$ (100 ps) time resolution in the vertexing (tracking) detector and only selecting hits in the narrow time window around the expected time of arrival, which is presented as a function of layer number in Fig. 2 (a). Tracking efficiency was evaluated by performing a detector simulation and track reconstruction in $H \rightarrow b \bar{b}$ events overlayed with the expected background particles. The resulting track-reconstruction efficiency as a function of track tranverse momentum is shown in Fig. 2 (b). The reduced tracking efficiency of the low-momentum tracks is caused by the limited number of iterations performed during the track reconstruction due to the contrained computing resources.

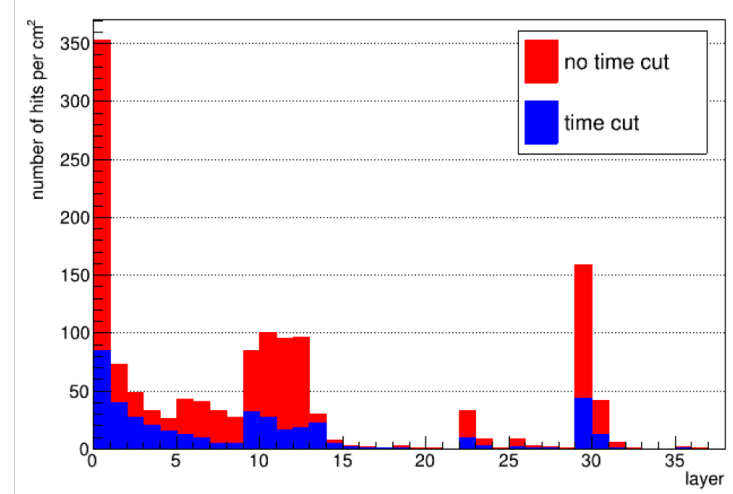

(a)

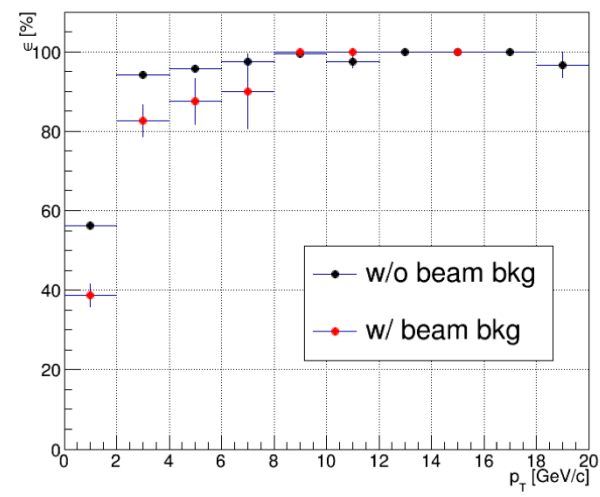

(b)

Figure 2: (a) Simulated occupancy as a function of tracker layer number before and after applying hit rejection outside the expected arrival time window. (b) Track-reconstruction efficiency in $H \rightarrow b \bar{b}$ events simulation with and without the beam-induced background.

Jet reconstruction was not included in the ILCRoot package, therefore a dedicated algorithm was developed using the reconstructed tracks and calorimeter clusters as input. A jet energy correction is applied as a function of the jet transverse momentum, and is determined by comparing 
the reconstructed jet energy to the energy of jets clustered from the Monte Carlo truth-level particles. The jet energy resolution was found to be $20 \%$ when no beam-induced background is present in the detector, and degraded to about $30 \%$ with the background included. The reconstructed dijet invariant mass in $H \rightarrow b \bar{b}$ events simulated with and without the beam-induced background is shown in Fig. 3. A significantly improved performance is expected with proper reconstruction of low-momentum tracks and an optimised jet clustering algorithm.
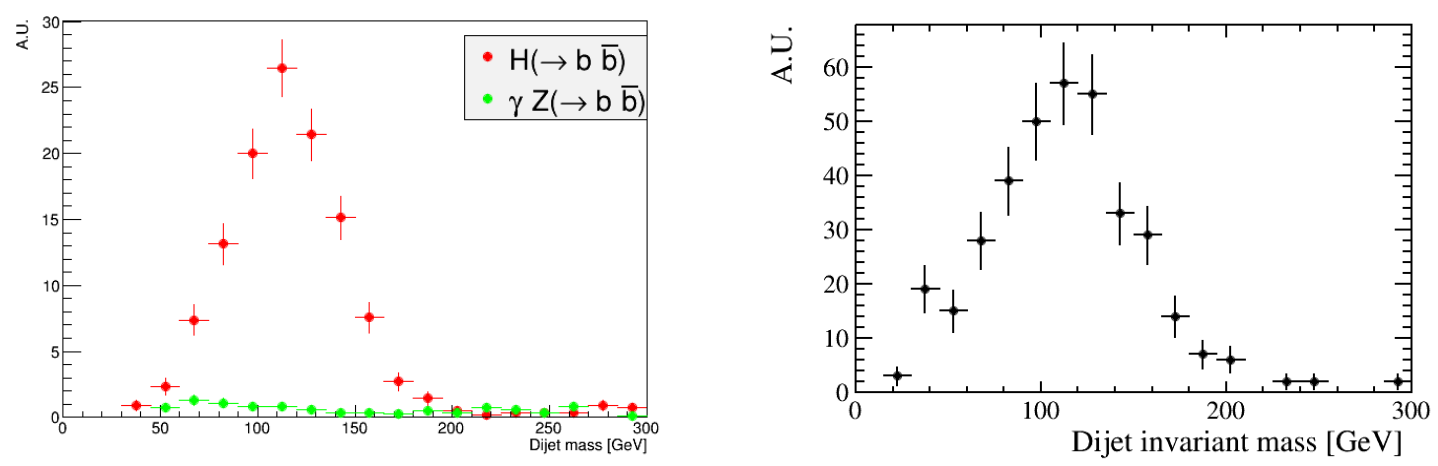

Figure 3: Reconstructed invariant mass of 2 jets in signal $H \rightarrow b \bar{b}$ events without (left) and with (right) the presence of the beam-induced background.

\section{Conclusions}

A preliminary study of the effects of the machine-induced background on the detector performance was carried out using the MAP simulation framework and MARS15 generated backgrounds for a muon collider with a centre-of-mass energy of $1.5 \mathrm{TeV}$. The detector performance is similar to results presented at conferences and more in-depth detector optimisation studies are in progress based on a modern software framework.

\section{References}

[1] J.P. Delahaye, M. Diemoz, K. Long, B. Mansoulié, N. Pastrone, L. Rivkin, D. Schulte, A. Skrinsky, A. Wulzer, "Muon Colliders", arXiv:1901.06150 [physics.acc-ph] (2019)

[2] The Muon Accelerator Program, https://map. fnal.gov/

[3] N.V. Mokhov and S.I. Striganov, "Detector Backgrounds at Muon Colliders", Phys. Procedia 37 (2012) [arXiv:1204.6721]

[4] N.V. Mokhov, S.I. Striganov, and I.S. Tropin, "Reducing backgrounds in the Higgs factory muon collider detector", arXiv:1409.1939 (2014)

[5] N.V. Mokhov and C.C. James, "The MARS Code System User's Guide Version 15 (2016)", FERMILAB-FN-1058-APC (2017)

[6] V. Di Benedetto et al., "A study of muon collider background rejection criteria in silicon vertex and tracker detectors", 2018 JINST 13 P09004

[7] C. Gatto et al., "Status of Dual-readout R\&D for a linear collider in T1015 Collaboration", arXiv:1603.00909 [physics.ins-det] (2016) 